\section{NEUROLOGICAL DISORDERS}

\section{The pull of the prions}

Misfolded prion protein causes the buildup of voltage-gated calcium channels inside the cell, interfering with normal neuronal communication and causing motor dysfunction, according to a recent study (Neuron 74, 300-313).

Mutations in the PRNP gene can lead to PrP protein aggregation and can cause a variety of neurological diseases in humans that are commonly linked to motor dysfunction. Assunta Senatore et al. found that, compared with mice expressing wild-type PrP, mice expressing mutant PrP showed reductions in glutamate release at synapses in the cerebellum, a part of the brain that controls motor function.

Voltage-gated calcium channels (VGCCs) are required at the surface of synapses for normal release of glutamate. The researchers found that mutant PrP bound VGCCs and prevented them from being delivered to the cell surface. These findings suggest that enhancing VGCC transport to synapses could ameliorate motor dysfunction in prion diseases. $-E C$

\section{IMMUNOLOGY}

\section{Bugs tune gut defenses}

Commensal bacteria colonize the gut mucosa, and secretion of IgA, the most abundant antibody type in mucosal secretions, by the gut is required to maintain immune homeostasis, promote tolerance and defend against pathogens in the presence of commensals.

A recent study now shows that the programmed cell death-1 (PD-1) inhibitory co-receptor regulates the microbiota by selecting the appropriate IgA repertoire (Science 336, 485-489). Using mice lacking PD-1, Kawamoto et al. found that its deficiency perturbed the bacterial composition. Although the number of IgA-producing cells in the lamina propria, the mucosal layer that recruits immune effector cells, stayed the same, there was a change in the IgA repertoire and less coating of bacteria by IgA in PD-1-deficient mice.

The altered IgA repertoire seen in PD-1deficient mice seems to occur because of an increased turnover of IgA-producing cells in the lamina propria, an effect that was abrogated after antibiotic treatment, suggesting that the microbiota drives selection of IgAs at this site. The authors also observed a more rapid transit of $B$ cells through germinal centers located in lymphoid structures, where antibody affinity maturation and diversification occur. Within germinal centers, $T$ follicular helper $\left(T_{F H}\right)$ cells

INFECTIOUS DISEASES

\title{
NO means yes to Listeria infection
}

Listeria monocytogenes

is a food-borne pathogen

that causes gastroenteritis

in healthy persons

and potentially fatal

systemic infections in

immunocompromised

individuals. The bacteria

are phagocytosed by

macrophages and

subsequently spread in a cell-to-cell manner that is crucial for their virulence. Caroline Cole et al. now shed

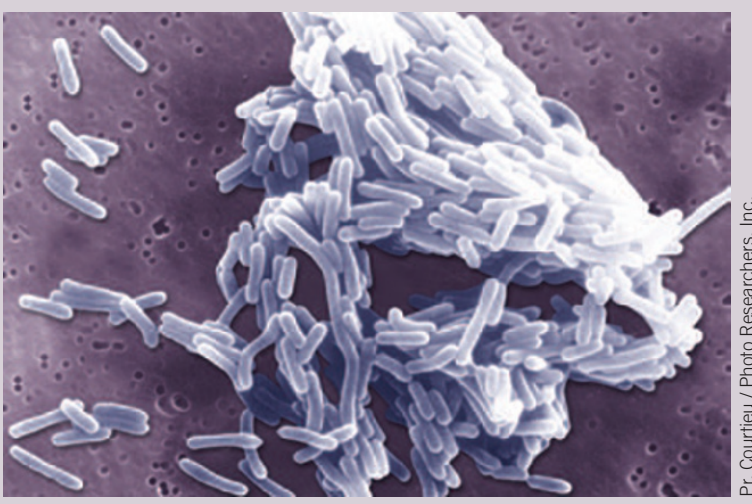

light on how $L$. monocytogenes avoids innate immune responses and subverts a host cell factor to facilitate its cell-to-cell spread. (Immunity doi:10.1016/j.immuni.2012.03.011).

Usually, the activation of Toll-like receptors by bacterial lipopolysaccharide (LPS) enhances the antimicrobial killing activity of macrophages, but the Gram-positive L. monocytogenes manages to avoid this fate. Cole et al. now show that even when macrophages are stimulated with LPS, their subsequent increased expression of nitric oxide synthase 2 (NOS2) and production of nitric oxide (NO) actually increases—rather than decreases-L. monocytogenes cell-to-cell spread.

Cell-to-cell spread of the bacteria occurs by the extrusion of pseudopods containing $L$. monocytogenes from a primary infected cell and their subsequent uptake by a recipient cell. Although the direct mechanism by which NO helps $L$. monocytogenes to spread remains unclear, the authors propose that NO delays the maturation of phagosomes that contain the bacteria, slowing their killing by the recipient cell. The researchers showed an increased escape of the bacteria into the cytosol associated with the delayed phagosome maturation and, ultimately, an increased bacterial burden. Understanding the mechanisms by which bacteria such as $L$. monocytogenes can circumvent immune responses may provide new insights into controlling the infections caused by these organisms. $-\boldsymbol{A F}$

help out B cells and promote their differentiation into antibody-secreting cells or memory $B$ cells. In the germinal centers of PD-1-deficient mice, $T_{F H}$ cells were more abundant and had a skewed cytokine profile, which may alter the selection of B cells and cause changes in the types of gut IgAs that they produce.

As mice lacking PD-1 showed hyperactivation of the immune system and production of antibodies against commensals, these findings suggest PD-1 absence may cause loss of tolerance. Further studies should investigate the role of PD-1 in autoimmune diseases in humans and its potential as a target to modulate these diseases. $-C P$

\section{METABOLIC DISORDERS}

\section{BuMPing up thermogenesis}

Brown adipose tissue (BAT) is coming to the forefront as a master regulator of metabolism and energy expenditure through its essential role in thermogenesis. A recent report provides clues on how this function is finely tuned by identifying bone morphogenetic protein 8B (BMP8B) as a regulator of thermogenesis that acts on both BAT and the central nervous system (Ce/l 149, 871-885).

BMP signaling has pleiotropic effects in many tissues, but, although BMPs have been shown to promote BAT differentiation and have an emerging role in adipogenesis, a role in BAT activation was not known. Whittle et al. now show that BMP8B is the prevalent BMP in mature BAT cells, where it is activated by prothermogenic stimuli and signaling.

In mice lacking BMP8B, the authors observed a progressive weight gain, which was exacerbated by a high-fat diet, and linked the fat accumulation to deficient BAT activation. Interestingly, BMP8B is also present in the regions of the hypothalamus known to control thermogenesis, and in its absence the brain could not properly respond to a high-fat diet by revving up BAT metabolism.

Treatment of mice with BMP8B induced sympathetic BAT activation through the brain and also caused an increased response to thermogenic signaling in BAT adipocytes, resulting in weight loss. Further studies will 